\title{
Recurrent nova IM Normae
}

\author{
T. Kato ${ }^{1}$, H. Yamaoka ${ }^{2}$, W. Liller ${ }^{3}$, and B. Monard ${ }^{4}$ \\ 1 Department of Astronomy, Kyoto University, Kyoto 606-8502, Japan \\ 2 Faculty of Science, Kyushu University, Fukuoka 810-8560, Japan \\ 3 Center for Nova Studies, Casilla 5022 Renaca, Viña del Mar, Chile \\ ${ }^{4}$ Bronberg Observatory, PO Box 11426, Tiegerpoort 0056, South Africa
}

Received 26 March 2002 / Accepted 2 July 2002

\begin{abstract}
We detected the second historical outburst of the 1920 nova IM Nor. Accurate astrometry of the outbursting object revealed the true quiescent counterpart having a magnitude of $R=17.0 \mathrm{mag}$ and $B=18.0 \mathrm{mag}$. We show that the quiescent counterpart shows a noticeable variation. From the comparison of light curves and spectroscopic signatures, we propose that IM Nor and CI Aql comprise a new class of recurrent novae bearing some characteristics similar to those of classical novae. We interpret that the noticeable quiescent variation can be a result of either high orbital inclination, or the presence of high/low states. If the second possibility is confirmed by future observations, IM Nor becomes the first recurrent nova showing state changes in quiescence. Such state changes may provide a missing link between recurrent novae and supersoft X-ray sources.
\end{abstract}

Key words. novae, cataclysmic variables - stars: individual: IM Nor

\section{Introduction}

IM Nor was originally discovered as a possible nova in 1920 by I. E. Woods from Harvard plates (cf. Elliot \& Liller 1972; Duerbeck 1987). The object was first detected on a plate taken on 1920 July 7 as a 9 mag star. Upon noting the possible identification with a UHURU X-ray source 2U 1536-52², Elliot \& Liller (1972) surveyed Harvard plates and constructed a light curve. Although the light curve was rather fragmentary, Elliot \& Liller (1972) suggested a similarity with the light curves of slow novae, especially DQ Her and the recurrent nova T Pyx. No spectroscopic observation was made during the 1920 eruption.

The quiescent identification of IM Nor was confusing. At the position of the nova, Elliot \& Liller (1972) remarked the presence of two stars near magnitude 21 . Wyckoff \& Wehinger (1979) (WW79) spectroscopically studied these stars and revealed that these stars are late-type stars without emission lines. Wyckoff \& Wehinger (1979) concluded that the star is a late-type companion of the nova, or that these stars are not physically associated with the nova. Wyckoff \& Wehinger (1979) set a lower limit of $\Delta B=11.7$ as the outburst amplitude. From this information and his original studies, Duerbeck (1987) (D87) reported the remeasured position of the nova,

Send offprint requests to: T. Kato,

e-mail: tkato@kusastro.kyoto-u.ac.jp

1 The proposed identification with $2 \mathrm{U} 1536-52=4 \mathrm{U} 1538-52$ was already questioned by Wyckoff \& Wehinger (1979). This X-ray source was later identified with an X-ray binary, QV Nor (see Kato 2002).
Table 1. Astrometry of IM Nor.

\begin{tabular}{|c|c|c|c|}
\hline \multirow[t]{2}{*}{ Source } & RA & Dec & \multirow{2}{*}{ Remarks } \\
\hline & \multicolumn{2}{|c|}{$(\mathrm{J} 2000.0)$} & \\
\hline WW79 & 153924 & -521934 & 1,2 \\
\hline D87 (Harvard plate) & 153926.25 & -521921.3 & 1 \\
\hline D87 (candidate 1) & 153926.12 & -521923.3 & 1 \\
\hline D87 (candidate 2) & 153926.50 & -521922.2 & 1 \\
\hline Liller (2002) & 153926.61 & -521918.6 & - \\
\hline Garradd (2002) & 153926.465 & -521917.99 & - \\
\hline This work & 153926.47 & -521918.2 & - \\
\hline Nearest USNO A2.0 & 153926.378 & -521918.66 & 3 \\
\hline DSS 2 counterpart & 153926.42 & -521917.9 & 4 \\
\hline
\end{tabular}

1: Precessed from B1950.0.

2: Remarked as incorrect in Duerbeck (1987).

3: Blended.

4: Marked with tick marks in Fig. 1.

and different candidates for the quiescent counterpart, whose exact identification remained uncertain (see Fig. 1).

The situation dramatically changed upon the discovery of the second historical outburst by W. Liller (Liller 2002). Subsequent observations confirmed the nova nature of the object (Duerbeck et al. 2002; Retter et al. 2002). This discovery qualifies IM Nor as the ninth recurrent nova in the Galaxy.

\section{Astrometry and identification of the Prenova}

Upon discovery of the outburst, we derived accurate astrometry from an unsaturated CCD image taken on 2002 January 


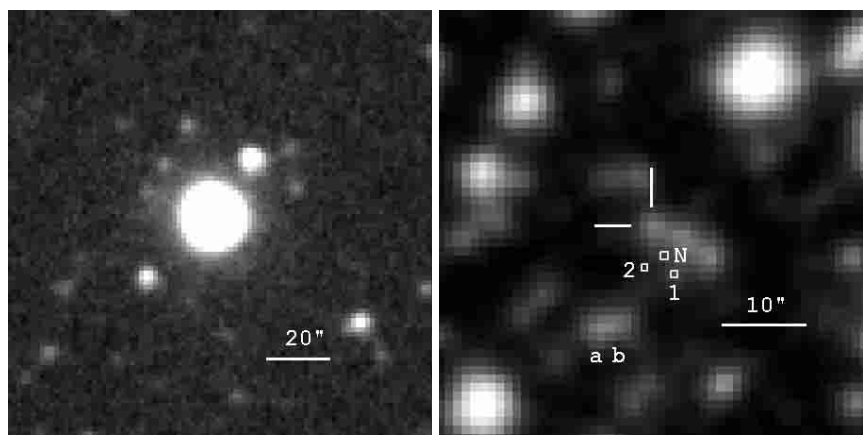

Fig. 1. Left: outburst image of IM Nor. Right: the quiescent counterpart of IM Nor (tick marks) on the DSS2 red image. The stars labeled a and b are the proposed counterparts by Elliot \& Liller (1972). The position of the nova and the proposed two candidate counterparts by Duerbeck (1987) are marked with squares labeled N, 1, 2, respectingly (see Table 1).
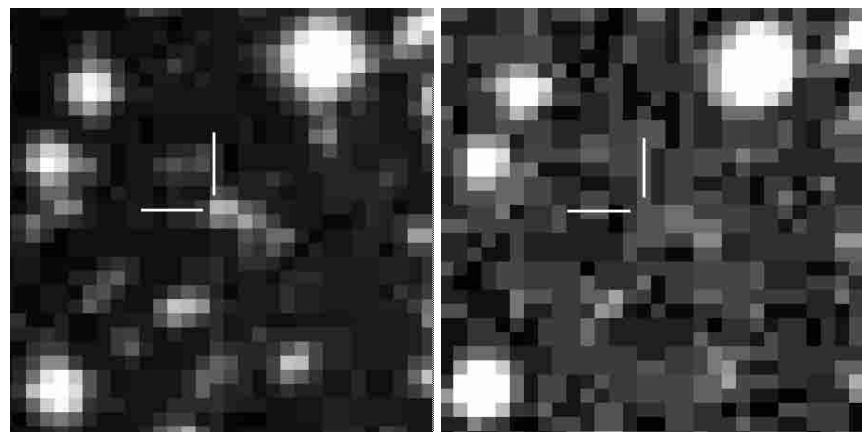

Fig. 2. Variation of the quiescent counterpart of IM Nor. Left: the $B$-band image taken on 1975 Jul. 7 with UK Schmidt (the DSS1). Right: the $V$-band image taken on 1987 Apr. 25 with the same telescope (also the DSS1). Also see Fig. 1 ( $R$ image, right).

15.104 UT with a 30-cm Schmidt-Cassegrain reflector at the Bronberg Observatory near Pretoria, South Africa. The astrometric reduction was done using 66 GSC-ACT stars (mean residual 0.3 ). Table 1 gives a summary of astrometry of this nova in the available literature.

The star is clearly different from the proposed candidates by Elliot \& Liller (1972) or by Duerbeck (1987; see Wyckoff \& Wehinger 1979). The correct identification and past suggested identifications are shown on a DSS2 red image in Fig. 1.

With an accurate position, we were able to identify IM Nor in several pre-outburst DSS images (Fig. 2). Using the APPHOT package in $\mathrm{IRAF}^{2}$ and the star measured by Wyckoff \& Wehinger (1979; $B=16.5$ for star to the SE) we obtained the prenova magnitudes listed in Table 2. The errors represent the photometric accuracies of the measurements on the linear part of the photographic response. These measurements show that the observed amplitude of the eruption was $\Delta B \sim 10$ mag and confirm Yamaoka's (2002) impression that the extreme colors $(B-V=-1.4, V-R=2.4)$ suggest quiescent variability. This effect was confirmed by performing aperture photometry on the neighboring constant stars.

${ }^{2}$ IRAF is distributed by the National Optical Astronomy Observatories.
Table 2. Photometry of quiescent IM Nor.

\begin{tabular}{cccc}
\hline \hline Plate & Date & Magnitude & Band \\
\hline J1628 & 1975 Jul. 7 & $18.0 \pm 0.3$ & $B$ \\
XV224 & 1987 Apr. 25 & $19.4 \pm 0.4$ & $V$ \\
XS224 & 1992 Jul. 24 & $17.0 \pm 0.3$ & $R$ \\
\hline
\end{tabular}

\section{Discussion}

\subsection{Classification among recurrent novae}

From the relatively blue quiescent color, the object is unlikely a symbiotic-type recurrent nova having a giant secondary (T CrB, RS Oph, V745 Sco, V3890 Sgr: for recent reviews of recurrent novae, see Sekiguchi 1995; Anupama \& Mikolajewska 1999; Hachisu \& Kato 2001). The object more resembles recurrent novae with main-sequence or slightly evolved secondaries (U Sco, V394 CrA, T Pyx, CI Aql and Nova LMC 1990 No. 2).

Among these objects, U Sco (Barlow et al. 1981; Sekiguchi et al. 1988), V394 CrA (Duerbeck 1988; Sekiguchi et al. 1989), and Nova LMC 1990 No. 2 (Sekiguchi et al. 1990) show extremely rapid declines, and are unlike IM Nor. As already suggested by Elliot \& Liller (1972), the slow decline of IM Nor resembles that of T Pyx. However, the light curve of the 2002 outburst (Fig. 3) drawn from observations reported to VSNET Collaboration ${ }^{3}$ more suggests a moderately fast nova with a $t_{3}$ of $\sim 50 \mathrm{~d}$. The moderately structured light curve, in contrast to those of fast recurrent novae (the best studied example being U Sco, see Hachisu et al. 2000a), also makes a resemblance to a recently recognized recurrent nova, CI Aql (Kiss et al. 2001; Matsumoto et al. 2001). From spectroscopy, IM Nor is reported to show long persistence of FeII lines (Retter et al. 2002) and later appearance of forbidden lines (Woodward et al. 2002), both suggesting that more material has been ejected than in a typical recurrent-nova outburst. The spectroscopic signature of massive ejecta makes a close resemblance to CI Aql which showed a classical novalike spectrum just after the maximum (Uemura \& Kato 2000) and the appearance of nebular lines at later stages (Matsumoto et al., in preparation). From these findings, we propose that IM Nor and CI Aql comprise a new subclass of recurrent novae with massive ejecta and long recurrence times.

\subsection{IM Nor in quiescence}

Quiescent IM Nor has several unique properties. As shown in Sect. 2, the total outburst amplitude of IM Nor is $\Delta B \sim$ $10.0 \mathrm{mag}$, indicating that the quiescent counterpart is fainter than usual recurrent novae (see e.g. Hachisu et al. 2000b; the large outburst amplitude of U Sco is difficult to reconcile without a special mechanism). Furthermore, IM Nor is unique among non-symbiotic recurrent novae in that it apparently shows significant variation in quiescence (Sect. 2; no high/low state transitions have been reported in T Pyx, CI Aql and U Sco). Such low quiescent brightness and the presence of

${ }^{3}$ http://www.kusastro.kyoto-u.ac.jp/vsnet/ 

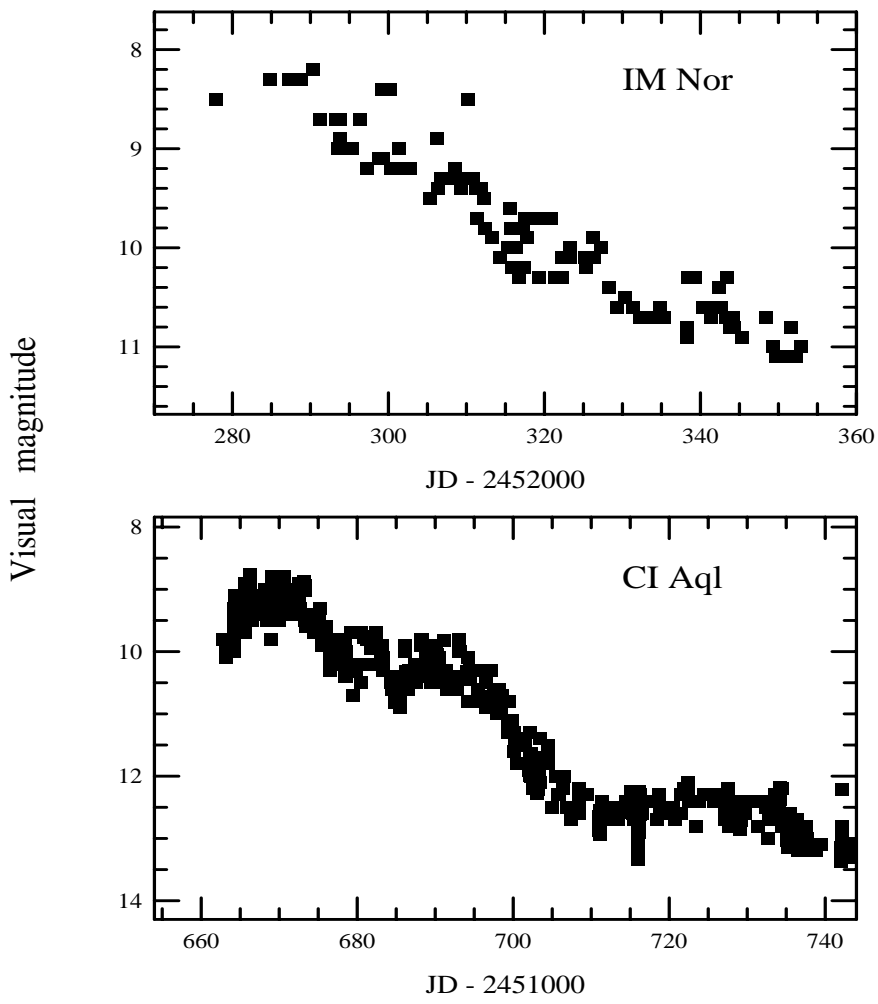

Fig. 3. Comparison of light curves of the 2002 outburst of IM Nor and the 2000 outburst of CI Aql. Modulations are superimposed on decays resembling those of moderately fast classical novae.

variations could be interpreted as an effect of a high inclination, i.e. the observed variation in quiescence may reflect eclipsetype or orbital variations. In such a case, one may expect to see orbital modulations or eclipses in near future (cf. U Sco: Hachisu et al. 2000a; CI Aql: Matsumoto et al. 2001).

Alternately, if no eclipses are observed in IM Nor, the presence of significant variation in quiescence makes the first indication of high/low states in (non-symbiotic type) recurrent novae. Since high/low state transitions are more commonly seen in supersoft X-ray source (the best example being RX J0527.8-6954: Greiner et al. 1996), which are supposed to be a close analog of recurrent novae (Kahabka et al. 1999; Hachisu et al. 1999), further observations and modeling of quiescent IM Nor is expected to provide a missing link between recurrent novae and supersoft X-ray sources.
Acknowledgements. The authors are grateful to the observers (Andrew Pearce, Jaime Garcia, Raquel Yumi Shida, Bruce Tregaskis, Alexandre Amorim), who reported visual observations of IM Nor to VSNET. This work is partly supported by a grant-in aid (13640239) from the Japanese Ministry of Education, Culture, Sports, Science and Technology. This research has made use of the Digitized Sky Survey producted by STScI, the ESO Skycat tool, and the VizieR catalogue access tool. The CCD operation of the Bronberg Observatory is partly sponsored by the Center for Backyard Astrophysics.

\section{References}

Anupama, G. C., \& Mikolajewska, J. 1999, A\&A, 344, 177

Barlow, M. J., Brodie, J. P., Brunt, C. C., et al. 1981, MNRAS, 195, 61 Duerbeck, H. W. 1987, Space Sci. Rev., 45, 1

Duerbeck, H. W. 1988, A\&A, 197, 148

Duerbeck, H. W., Baptista, R., Dutra, C. M., \& Sterken, C. 2002, IAU Circ., 7799

Elliot, J. L., \& Liller, W. 1972, ApJ, 175, L69

Garradd, G. J. 2002, IAU Circ., 7796

Greiner, J., Schwarz, R., Hasinger, G., \& Orio, M. 1996, A\&A, 312, 88

Hachisu, I., \& Kato, M. 2001, ApJ, 558, 323

Hachisu, I., Kato, M., Kato, T., \& Matsumoto, K. 2000a, ApJ, 528, L97

Hachisu, I., Kato, M., Kato, T., Matsumoto, K., \& Nomoto, K. 2000b, ApJ, 534, L189

Hachisu, I., Kato, M., Nomoto, K., \& Umeda, H. 1999, ApJ, 519, 314

Kahabka, P., Hartmann, H. W., Parmar, A. N., \& Negueruela, I. 1999, A\&A, 347, L43

Kato, T. 2002, IAU Circ., 7791

Kiss, L. L., Thomson, J. R., Ogloza, W., Furész, G., \& Sziládi, K. 2001, A\&A, 366, 858

Liller, W. 2002, IAU Circ., 7789

Matsumoto, K., Uemura, M., Kato, T., et al. 2001, A\&A, 378, 487

Retter, A., O'Toole, S. J., \& Starrfield, S. 2002, IAU Circ., 7818

Sekiguchi, K. 1995, Ap\&SS, 230, 75

Sekiguchi, K., Caldwell, J. A. R., Stobie, R. S., \& Buckley, D. A. H. 1990, MNRAS, 245, 28

Sekiguchi, K., Catchpole, R. M., Fairall, A. P., et al. 1989, MNRAS, 236, 611

Sekiguchi, K., Feast, M. W., Whitelock, P. A., et al. 1988, MNRAS, 234, 281

Uemura, M., \& Kato, T. 2000, IAU Circ., 7409

Woodward, C. E., Miller, B., \& Starrfield, S. G. 2002, IAU Circ., 7896

Wyckoff, S., \& Wehinger, P. A. 1979, PASP, 91, 173

Yamaoka, H. 2002, IAU Circ., 7791 